\title{
Generation of Energetic Electrons during Spherical Tokamak Merging in UTST ${ }^{*}$
}

\author{
Tomohiko USHIKI, Michiaki INOMOTO, Kotaro YAMASAKI, Xuehan GUO, \\ Takumichi SUGAWARA, Keita MATSUYAMA, Haruhisa KOGUCHI ${ }^{1)}$ and Takuma YAMADA ${ }^{2)}$ \\ Graduate School of Frontier Sciences, The University of Tokyo, Kashiwa 277-8656, Japan \\ ${ }^{1)}$ National Institute of Advanced Industrial Science and Technology, Tsukuba 305-8586, Japan \\ ${ }^{2)}$ Facility of Arts and Science, Kyushu University, Fukuoka 819-0395, Japan
}

(Received 30 November 2015 / Accepted 2 June 2016)

\begin{abstract}
Bursts of bremsstrahlung soft X-ray (SXR) emission $(>200 \mathrm{eV})$ were observed during spherical tokamak merging in the University of Tokyo Spherical Tokamak (UTST) experiment. SXR signal waveforms coincided well with the time evolution of a reconnection electric field, and their intensity shows a clear dependence on the strength of a toroidal magnetic field. This result suggests that electrons near the X-point are effectively accelerated in the toroidal direction by a parallel electric field during plasma merging in the presence of a strong toroidal magnetic field.
\end{abstract}

(c) 2016 The Japan Society of Plasma Science and Nuclear Fusion Research

Keywords: spherical tokamak, plasma merging, energetic electron, magnetic reconnection

DOI: $10.1585 /$ pfr. 11.2402100

\section{Introduction}

A Spherical tokamak (ST) [1] is a variant of a tokamak with a small aspect ratio, generally less than 2. An ST can satisfy both good confinement and high beta value, which is defined by the ratio of the plasma thermal pressure to magnetic pressure, and is expected to provide economical fusion core plasma for power plants and neutron sources. Nevertheless, the start-up scenario of ST plasma in a reactor has not yet been well established because it is not advantageous to employ conventional inductive current ramp-up schemes using a central solenoid (CS) coil. Because the small aspect ratio of an ST reduces the available space in the central region of a vacuum vessel, the CS coil significantly restricts the flexibility of the reactor design. Therefore, the development of a CS-less start-up method is one of the most important issues facing ST reactors.

Various current drive schemes such as an RF current drive $[2,3]$ and helicity injection $[4,5]$ are under development in several ST experiments performed worldwide. Another CS-less start-up method is employed in START, MAST (UKAEA) [6], and TS-3/TS-4 [7, 8] devices using induction from in-vessel PF coils as well as in the UTST device [9] using induction from ex-vessel PF coils in combination with plasma merging, which can provide high-power initial heating via magnetic reconnection and density increases via compression. Recent experiments in a MAST device [10] provided detailed measurements of ion and electron heating during plasma merging. Although downstream ion heating during magnetic reconnection has

author'se-mail: ushiki@ts.t.u-tokyo.ac.jp

*) This article is based on the presentation at the 25th International Toki Conference (ITC25). been quantitatively evaluated, electron heating (or energization) near the $\mathrm{X}$-point in collisionless reconnection is not yet well understood. Similar local electron heating has also been observed in UTST experiments [11], and it was concluded that the thermalization of energetic electrons accelerated by a reconnection electric field along a guide (toroidal) magnetic field perpendicular to a reconnection (poloidal) magnetic field can account for local electron heating.

Parallel acceleration near the X-point and separatrix region of magnetic reconnection with a guide field has also been predicted by numerical studies $[12,13]$; however, no experimental verification has been conducted. Indirect evidence of electron acceleration in the UTST device has been obtained. Figure 1 (right) shows a fast camera image clicked during the merging period. A ring-shaped emission of an impurity line appeared near the X-point and exhibited a highly elongated structure in the toroidal direction, implying that fast electrons were generated via direct acceleration by a reconnection electric field along a toroidal magnetic field. In this study, the mechanism of parallel electron acceleration during guide field reconnection in the UTST device is investigated by via soft X-ray (SXR) measurements.

\section{Experimental Setup}

The goal of the UTST merging experiment is to demonstrate an ST start-up scheme using only an ex-vessel PF coil swing. Figure 2 shows a schematic of the merging start-up method: (a) two initial STs are generated by the inductive electric field supplied via the PF coil swing; (b) 

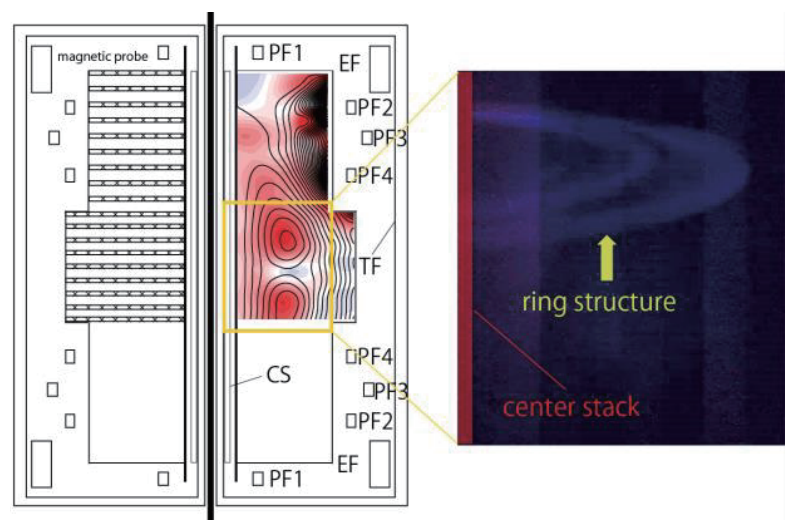

Fig. 1 Cross-sectional view of UTST device and fast-camera image (CIII: $467 \mathrm{~nm}$ ) of reconnection region during ST merging.

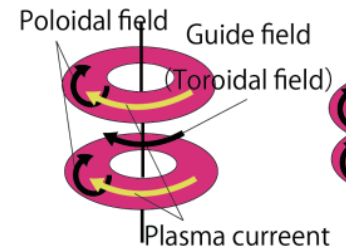

(a)

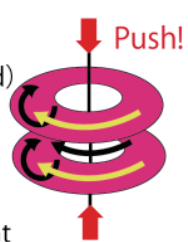

(b)

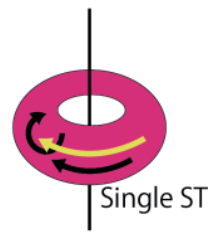

(c)
Fig. 2 Schematic of merging start-up method.

Table 1 Typical parameter for the ST merging start-up.

\begin{tabular}{lc}
\hline Chamber radius & $\begin{array}{c}0.7 \mathrm{~m} \text { (midplane) } \\
0.6 \mathrm{~m} \text { (top \& bottom) }\end{array}$ \\
\hline Chamber height & $2.0 \mathrm{~m}$ \\
\hline Toroidal field (Guide field) & $0.1-0.3 \mathrm{~T}$ \\
\hline Poloidal field (Reconnection field) & $0.01-0.02 \mathrm{~T}$ \\
\hline Major radius & $0.45 \mathrm{~m}$ \\
\hline Aspect ratio & 1.3 \\
\hline Plasma current & $130 \mathrm{kA}(\mathrm{w} / \mathrm{o} \mathrm{CS})$ \\
\hline Ion temperature & $200 \mathrm{kA}($ with CS$)$ \\
\hline Electron temperature & $\sim 50 \mathrm{eV}$ \\
\hline Electron density & $10-30 \mathrm{eV}$ \\
\hline
\end{tabular}

the formed STs are pushed toward the midplane; and (c) finally, the two STs merge to form a single ST via magnetic reconnection. Typical parameters of the UTST merging experiment are presented in Table 1.

A two dimensional pick-up coil array is employed in the UTST device to directly measure the internal magnetic field profile. The poloidal magnetic flux, current density, and toroidal electric field are calculated using Eqs. (1), (2), and (3), respectively, as follows:

$$
\begin{aligned}
& \Psi(R, Z, t)=2 \pi \int_{r_{0}}^{R} B_{z}(\rho, Z, t) \rho \mathrm{d} \rho, \\
& j_{t}(R, Z, t)=-\frac{1}{\mu_{0}}\left(\frac{1}{2 \pi R} \frac{\partial^{2} \Psi(R, Z, t)}{\partial Z^{2}}+\frac{\partial B_{z}(R, Z, t)}{\partial R}\right), \\
& E_{t}(R, Z, t)=-\frac{1}{2 \pi R} \frac{\mathrm{d} \partial(R, Z, t)}{\mathrm{d} t} .
\end{aligned}
$$

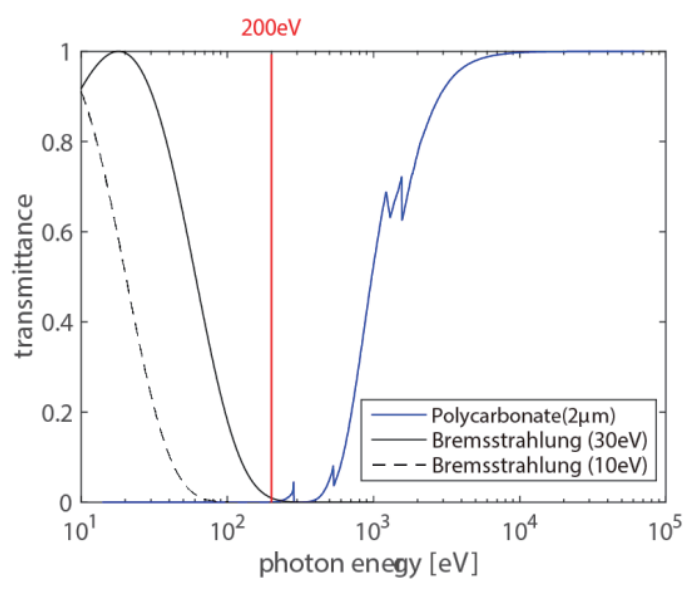

Fig. 3 Transmittance of polycarbonate X-ray absorption filter by data of NIST [15]. Dashed and solid black lines show thermal bremsstrahlung from the electrons of $10 \mathrm{eV}$ and $30 \mathrm{eV}$, respectively.

Because the poloidal magnetic field reconnects during plasma merging in the UTST experiment, the induced reconnection electric field is in the toroidal direction. Therefore, in the ST merging experiment, in which a strong toroidal field is applied by an external coil, the reconnection electric field becomes field aligned near the X-point where the poloidal field is negligible [14]. The typical ratio of the toroidal (guide) field to poloidal (reconnection) field is $10-20$ in the UTST experiment, which is much higher than those in previous TS-3/4 experiments or numerical studies. This is unique features of the UTST merging experiment that can provide remarkable electron acceleration near the X-point.

A surface barrier detector (SBD: Ortec model CR017-050-100) was employed to observe SXR emission from electron bremsstrahlung during magnetic reconnection. The SBD is a diode covered with a thin aluminum $(0.15 \mu \mathrm{m})$ rectifying contact on $n$-type $\mathrm{Si}$ with good sensitivity over the energy range of SXR $(<20 \mathrm{keV})$. The dead layer of $\mathrm{Si}$ is $0.01 \mu \mathrm{m}$. SXR from the reconnection $\mathrm{X}$ point region are detected by the SBD, which has a tangential line-of-sight with a radius of $35 \mathrm{~cm}$. This radius is nearly the same as the $\mathrm{X}$-point radius. A pinhole is placed in front of the SBD to adjust the spot size of the viewing field to cover the entire reconnection region. A polycarbonate $(2 \mu \mathrm{m})$ foil was employed as an X-ray absorption filter to detect SXR ( $>200 \mathrm{eV})$. The total transmittance including the absorption effects of rectified aluminum and the Si dead layer, is shown in Fig. 3. The current detected by the SBD is converted into a voltage signal using an I/V converter and amplified using a preamplifier.

\section{Experimental Results}

Figure 4 shows the typical waveforms of reconnection parameters such as (upper) the reconnected magnetic flux, (middle) the reconnection electric field, and (bot- 


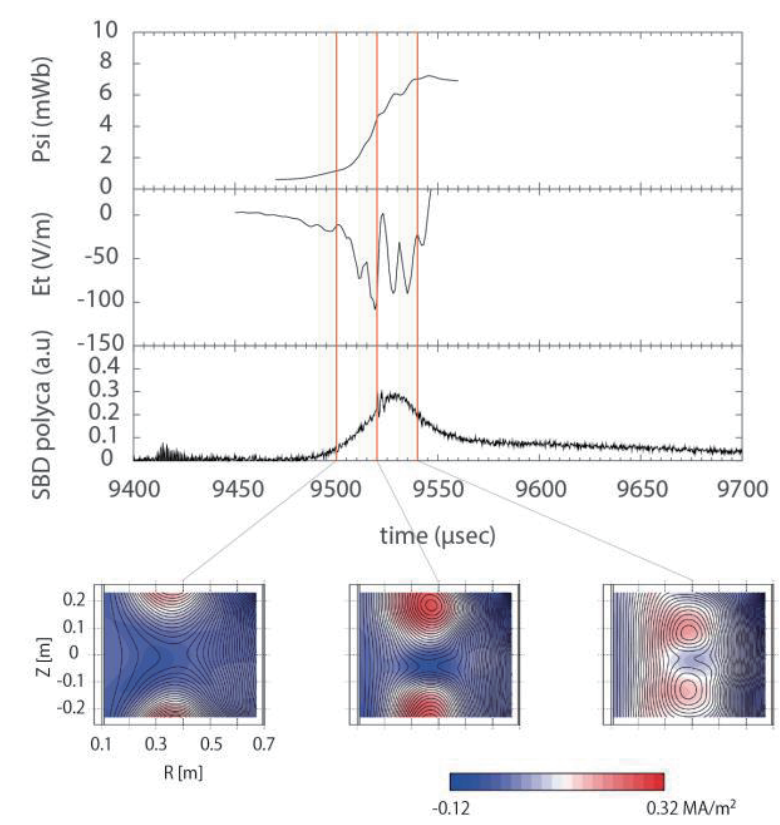

Fig. 4 Evolution of reconnection parameters (upper) reconnection magnetic flux, (middle) toroidal current density, and (bottom) soft X-ray (polycarbonate: $2 \mu \mathrm{m}$ ) during reconnection. Corresponding poloidal flux surfaces are also shown.

tom) the soft X-ray signal during magnetic reconnection and their corresponding poloidal flux surfaces. Sharp SXR (>200 eV) bursts were observed only during magnetic reconnection with a roughly good correlation with the low frequency behavior of the reconnection electric field waveform at the X-point. There are two possible origins of the SXR bursts: thermal and non-thermal electrons generated during reconnection. The Thomson scattering measurement revealed that the electron temperature in the UTST merging experiment was up to $30 \mathrm{eV}$ [11], which is much lower than the absorption edge of the filter used in the present study, as shown in Fig. 3. Therefore, it is believed that non-thermal fast electrons accelerated by the fieldaligned electric field account for the observed SXR.

The intensities of the SXR bursts were affected by both the reconnection electric field and toroidal guide field in the UTST merging experiment. Figure 5 shows the dependence of the SXR intensity on $E_{\mathrm{t}}\left(B_{\mathrm{t}} / B_{\mathrm{p}}\right)$ assuming. The SXR intensities exhibited a clear increasing trend with $E_{\mathrm{t}}\left(B_{\mathrm{t}} / B_{\mathrm{p}}\right)$, suggesting that the efficiency of electron acceleration can be expressed via the effective electric field $E_{\text {eff }} \sim E_{\mathrm{t}}\left(B_{\mathrm{t}} / B_{\mathrm{p}}\right)$ near the $\mathrm{X}$-point region in analogy with torus plasma breakdown, where electrons near the null point are effectively accelerated [16].

\section{Discussion}

Based on the experimental results presented above, possible mechanisms to generate energetic electrons are now discussed. To quantitatively evaluate electron acceleration, a simple OD model considering the acceleration

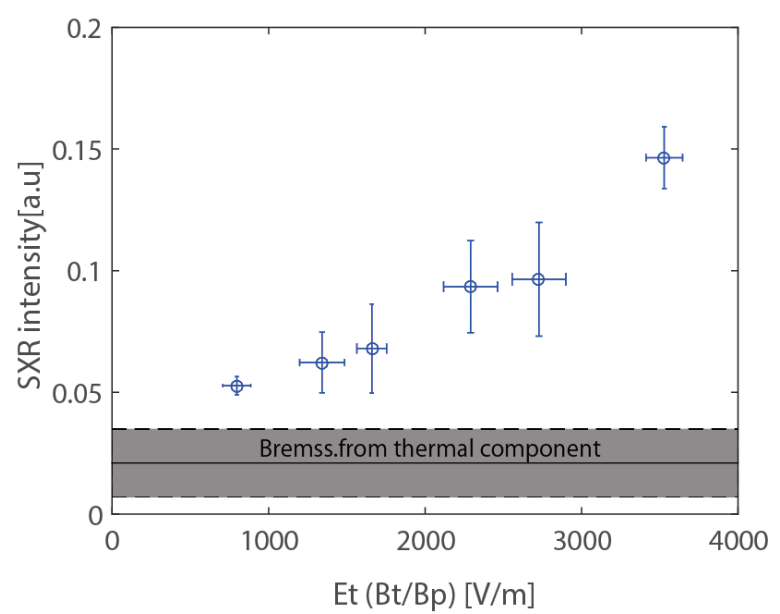

Fig. 5 Dependence of soft X-ray intensity on $E_{\text {eff }}$ at point $B_{\mathrm{p}}=$ $0.01 \mathrm{mT}$.

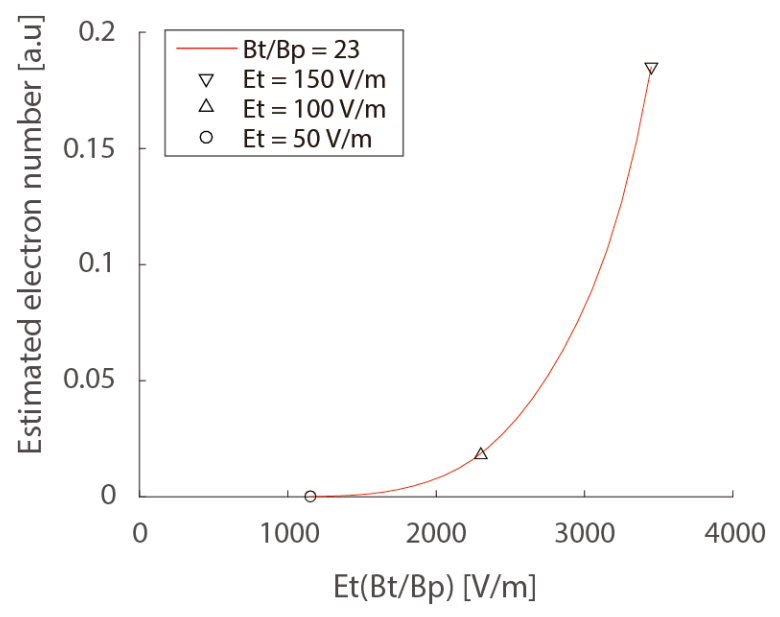

Fig. 6 Dependence of estimated electron number, including absorption effect of polycarbonate $(2 \mu \mathrm{m})$ filter, on $E_{\text {eff }}$.

period was developed. In this model, electrons are accelerated along the total magnetic field $B_{\mathrm{t}}+B_{\mathrm{p}}$. Because an acceleration region is limited by the half length of a reconnection region, the electrons can stay inside within the period while their travel length injected to poloidal plane stay inside $L$, i.e., the total travel length is defined as $L_{\mathrm{t}}=L\left(B_{\mathrm{t}}^{2}+B_{\mathrm{p}}^{2}\right)^{1 / 2}$. We assume here that Coulomb collisions act as a breaking force. In this manner, the acceleration period can be derived based on the magnetic field angle at the X-point.

The experimental conditions were $B_{\mathrm{t}} / B_{\mathrm{p}}, E_{\mathrm{t}}=50 \sim$ $150 \mathrm{~V} / \mathrm{m}, n_{\mathrm{i}}=2 \times 10^{19}, B_{\mathrm{p}}=0.01 \mathrm{mT}$, initial electron temperature $T_{\mathrm{e}}=10 \mathrm{eV}$, and $L=0.1 \mathrm{mT}$. Figure 6 shows the dependence of the estimated electron number by considering the absorption effect of the polycarbonate $(2 \mu \mathrm{m})$ filter on the effective electric field $E_{\text {eff }}$. The estimated electron number is described as

$$
\text { Estimated electron number } \propto \int T(E) f(E) \mathrm{d} E,
$$


where $E, T(E)$, and $f(E)$ are the photon energy, total transmittance (including the absorption effect of rectified aluminum and the Si dead layer, as shown in Fig. 3), and electron velocity distribution function caluclated using the motion equation, $E_{\text {eff }}$, and initial conditions, respectively. Several electrons were accelerated to over $200 \mathrm{eV}$ during their stay inside the reconnection region. The calculation result shows that the SXR signal appears when $E_{\text {eff }}$ exceeds approximately $1000 \mathrm{~V} / \mathrm{m}$, which is similar to the experimental results; however, a relatively steeper increasing trend was predicted using the numerical method. This result qualitatively agrees with the experimental results shown in Fig. 5.

\section{Conclusions}

To investigate the electron acceleration mechanism during high guide field magnetic reconnection, experimental observations of soft X-ray measurement in UTST plasma merging device were carried out. The results of this study are as follows.

- High energy SXR ( $>200 \mathrm{eV}$ ) were detected only during merging.

- The peak intensity of SXR showed a positive dependence on $E_{\text {eff }}$.

- A 0D model partly explains the dependence of the number of electrons over $200 \mathrm{eV}$ on $E_{\text {eff }}$.

From these results, local acceleration near the X-point in a direction parallel to a magnetic field is believed to ac- count for the generation of energetic electrons over $200 \mathrm{eV}$.

\section{Acknowledgments}

This work was supported by the JSPS A3 Foresight Program "Innovative Tokamak Plasma Startup and Current Drive in Spherical Torus", Grants-in Aid for Scientific Research (KAKENHI) 15H05750, 15K14279, 26287143, and 25820434, and the NIFS Collaboration Research program(NIFS14KNWP004). The authors would like to thank Enago (www.enago.jp) for the English language review.

[1] Y.-K.M. Peng, Phys. Plasmas 7, 1681 (2000).

[2] K. Hanada et al., Plasma Fusion Res. 5, S1007 (2010).

[3] T. Wakatsuki et al., Nucl. Fusion 54, 093014 (2014).

[4] R. Raman et al., Nucl. Fusion 41, 1081 (2001).

[5] N.W. Eidietis et al., J. Fusion Energy 26, 43 (2007).

[6] Y. Ono et al., Plasma Phys. Control. Fusion 54, 124039 (2012).

[7] Y. Ono et al., Phys. Rev. Lett. 76, 3328 (1996).

[8] E. Kawamori et al., Phys. Rev. Lett. 95, 085003 (2005).

[9] M. Inomoto et al., Nucl. Fusion 55, 033013 (2015).

[10] H. Tanabe et al., Phys. Rev. Lett. 115, 215004 (2015).

[11] X. Guo et al., Phys. Plasmas 22, 101201 (2015).

[12] P.L. Pritchett and F.V. Coroniti, J. Geophys. Res. 109, A01220 (2004).

[13] P. Ricci et al., Phys. Plasmas 11, 4102 (2004).

[14] K. Yamasaki et al., Phys. Plasmas 22, 101202 (2015).

[15] NIST website (http://www.nist.gov/pml/data/asd.cfm)

[16] B. Lloyd et al., Nucl. Fusion 31, 2031 (1991). 